\title{
Speed-accuracy tradeoff in double stimulation: II. Effects on the second response
}

\author{
JAMES L. KNIGHT, JR. and BARRY H. KANTOWITZ \\ Purdue University, West Lafayette, Indiana 47907
}

\begin{abstract}
In the double-stimulation paradigm subjects respond to two successive stimuli. Previous research (Knight \& Kantowitz, 1974) showed that a subject's speed-accuracy tradeoff (SAT) strategy interacted with the interval between the two stimuli to determine response performance to the first stimulus. The present experiment examined the influence of SAT strategy on response performance to the second stimulus. Interest focused on effects of SAT strategy upon the psychological refractory period (PRP) effect. If a single mechanism underlies both first- and second-response performance (e.g., the PRP effect) in double stimulation, effects of SAT upon the second response should be similar to effects upon the first response. Results showed that the PRP effect appeared only when second-response accuracy was stressed. Under speed emphasis double-stimulation second-response latency never exceeded a single-stimulation baseline. This was analogous to first-response latency effects found by Knight and Kantowitz (1974). Response grouping was strongly influenced by SAT strategy and two response-grouping mechanisms were distinguished. Implications of these and interresponse time data for models of double-stimulation performance are discussed.
\end{abstract}

In double stimulation two stimuli are presented sequentially. Often the response to the second stimulus is found to be delayed when compared to responses to the same stimulus presented alone. The delay above a single-stimulation baseline usually increases as the interstimulus interval (ISI) separating onsets of first $\left(\mathrm{S}_{1}\right)$ and second $\left(\mathrm{S}_{2}\right)$ stimuli decreases (Herman \& Kantowitz, 1970). The inverse relationship between secondresponse latency and ISI is referred to as the psychological refractory period (PRP) effect. Interest in this effect is based on the likelihood that it results from a basic sequential processing limitation with strong implications for models of attention (Kantowitz, 1974a).

In a previous paper (Knight \& Kantowitz, 1974), the present authors examined first-response $\left(R_{1}\right)$ performance in two double-stimulation paradigms since, ironically, extant theories of second-response $\left(\mathbf{R}_{2}\right)$ performance (i.e., the PRP effect) can best be tested by their predictions concerning $R_{1}$ (Herman \& Kantowitz, 1970). In the earlier paper Knight and Kantowitz (1974) found that $R_{1}$ performance consistently declined as ISI decreased, in a manner analogous to the PRP effect; however, the form of this $R_{1}$ decrement (increased latency or increased errors) depended upon the speed-accuracy tradeoff (SAT) strategies adopted by subjects.

The SAT strategy refers to the relative emphases placed upon response speed and accuracy. Usually the

This research was supported by NIMH Grant MH-26302. We would like to acknowledge the insightful review and helpful criticisms provided by C. R. R. Snyder on an earlier version of this paper. Requests for reprints should be sent to James $L$. Knight, Jr., Department of Psychological Sciences, Purdue University, West Lafayette, Indiana 47907. choice of strategy reflects the explicit or implicit costs of time and errors in a task since, at a particular level of practice, subjects are unable to simultaneously maximize both speed and accuracy and must sacrifice one for the other. Consequently, Knight and Kantowitz (1974) concluded that variation in SAT strategies could account for much of the apparently conflicting data concerning the question of $R_{1}$ latency increases with decreasing ISI. As discussed by Herman and Kantowitz (1970) and by Knight and Kantowitz (1974), the finding of consistent $R_{1}$ performance decrements, whether manifested as increased latency or inaccuracy, supports a responseconflict model of double stimulation.

Since the previous experiment (Knight \& Kantowitz, 1974) was designed only to evaluate $R_{1}$ effects, it was unsuited for examination of the PRP and other $R_{2}$ effects. The principal difficulty was that the conditional uncertainty associated with $S_{2}$ given that $S_{1}$ had already appeared was zero: Stimuli could appear in one of two locations with two response keys spatially mapped to them. While $S_{1}$ could appear in either location, and thus involved 1 bit of uncertainty, $S_{2}$ could appear only in the location not occupied by $S_{1}$. Thus, inferences concerning $S_{2}$ processing were severely constrained. In particular, it was not possible to draw independent inferences concerning the accuracy of $R_{2}$ and subjects' abilities to trade $R_{2}$ accuracy for increased speed. Also the lack of conditional $S_{2}$ event uncertainty, coupled with a blocked-ISI procedure, increased the possibility that subjects would not process $S_{2}$ at all but would merely execute a "grouped" response pair in which $R_{2}$ was timed to occur at a fixed interval (corresponding to the ISI in effect) after $R_{1}$.

The present experiment was more appropriately designed to assess SAT effects upon $R_{2}$ (and especially 
upon the PRP effect) by (1) separating $S_{1} \cdot R_{1}$ and $S_{2} \cdot R_{2}$ sets and (2) providing equal event uncertainties (1 bit) for both $S_{1} \cdot R_{1}$ and $S_{2}-R_{2}$. While $R_{1}$ latency increments have been found only inconsistently, the PRP effect is ubiquitous. Thus, while manipulation of SAT strategies eliminated $R_{1}$ latency increments, suggesting a degree of subject control over such performance decrements, the PRP effect might not be so susceptible to the subject's SAT strategy. Keele (1973), for example, has suggested that the PRP effect reflects a basic minimum delay which must separate successive response initiations. This delay is not dependent upon the level of processing used to determine which response should be initiated. Such a limitation would probably be unaffected by a subject's cognitive SAT strategy.

While the present paradigm was designed to allow examination of $R_{2}$ performance, it also permits analysis of $R_{1}$ effects. These effects are of interest for three reasons. First, the nature of $R_{1}$ effects may help to clarify the mechanisms underlying $R_{2}$ results. For example, the single-channel model (Welford, 1952) of double-stimulation performance suggests that $R_{2}$ delays will be obtained to the extent that $S_{2}$ occurs before $R_{1}$ (i.e., $\mathrm{RT}_{1}<\mathrm{ISI}$ ).

Second, the present $R_{1}$ results can be compared to those of Knight and Kantowitz (1974). This is particularly appropriate since in both studies the first stimulus involved 1 bit of uncertainty. Differences between these experiments include overlap of first and second stimulus and response sets (i.e., on different trials, a particular stimulus and response might serve as $S_{1}$ and $R_{1}$ or as $S_{2}$ and $R_{2}$ ) in the former, but not in the present, study.

Third, because both $S_{1}$ and $S_{2}$ involve 1 bit of event uncertainty and are drawn from nonoverlapping sets, the present experiment offers a further test of the unitary-mechanism assumption that is utilized by response-conflict and other models of doublestimulation performance (Herman \& Kantowitz, 1970; Kahneman, 1973; Kantowitz, 1974b; Reynolds, 1964). According to this unitary assumption, a single process underlies both $R_{1}$ and $R_{2}$ double-stimulation effects. Thus, generally, $R_{1}$ and $R_{2}$ performance should be affected by experimental variables in similar ways. Variants of this unitary assumption are also embodied in Triggs' (Note 1) capacity-sharing model and Kahneman's (1973) variable-allocation model of double stimulation. A second, independent assumption of response-conflict theory is that this single process is localized in response- rather than stimulus-processing stages (Kantowitz, 1974a, b).

Finally, the present experiment sought to examine effects of an intermediate payoff condition in which both speed and accuracy of performance were given equal importance. This condition was included to determine if performances observed under speed-stress and accuracy-stress payoffs represented points on a continuum along which performance under the equalpayoff condition would fall or, alternatively, represented the only two processing modes between which subjects could choose. In this case, performance under the equalpayoff condition might be highly variable, sometimes resembling that produced under speed emphasis and at other times resembling that obtained under accuracy emphasis.

\section{METHOD}

\section{Subjects}

Eighteen right-handed female volunteers served as subjects in exchange for course credit in introductory psychology. Subject-use guidelines of the American Psychological Association were followed.

\section{Apparatus}

A PDP-8/L computer equipped with a crystal time-base clock was used to control stimulus presentation, data recording, and temporal sequencing. The computer-controlled clock permitted latency measurement accuracy within $1 \mathrm{msec}$. Stimuli were presented on a Tektronix Model $5103 \mathrm{~N}$ display scope equipped with a high-intensity P31 phosphor.

\section{Design}

Six subjects were randomly assigned to each of three experimental groups. These groups (speed, neutral, accuracy) were distinguished by the speed vs accuracy payoff matrices (Figure 1) used to score responses. Two of these payoff matrices (speed and accuracy) were previously utilized by Knight and Kantowitz (1974). Each subject was tested on six blocks of 40 discrete trials and each block was characterized by a different constant ISI $(30,60,120,240 \mathrm{msec}$, or single-stimulation control) separating onsets of $S_{1}$ and $S_{2}$. Within each group of six subjects, ISI presentation order was counterbalanced according to a 6 by 6 digram-balanced Latin Square.

The 40 trials of each block consisted of 32 experimental trials preceded by eight practice trials. In the practice trials of double-stimulation blocks, each of four possible $S_{1}$ by $S_{2}$ combinations occurred twice and each possible foreperiod $(\mathrm{FP}=1.5,1.75,2.25$, or $2.5 \mathrm{sec})$ separating onsets of a warning signal and $S_{1}$ also occurred twice. In the eight practice trials of the single-stimulation block, the two possible $S_{1} s$ each occurred alone four times and each possible FP occurred twice.

In the 32 experimental trials of each double-stimulation block, each of 16 possible trial events occurred twice in one of five random sequences. The 16 trial events were formed by factorial combination of two possible $S_{1} s$, two possible $S_{2} s$, and four possible FPs. For the single-stimulation control block
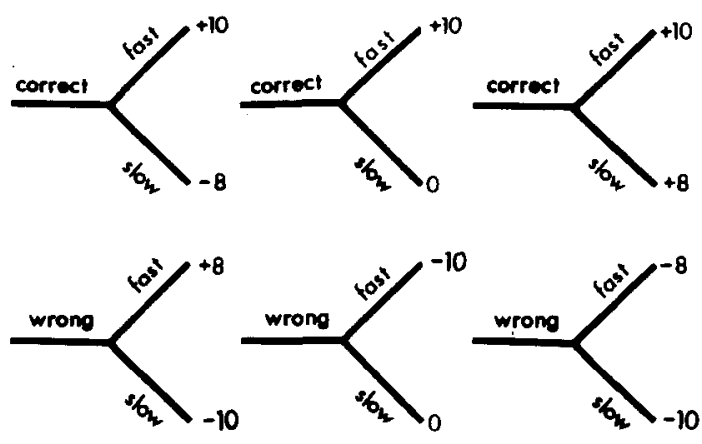

Speed Noutral Accuracy

Figure 1. Payoff matrices used to score responses. 
there were only eight possible trial events. These were formed by combination of two possible $S_{1} s$ with four possible FPs. Each of the eight trial events was presented four times in a randomly ordered experimental-trial series.

\section{Procedure}

Subjects were tested individually in a dim, quiet room. While in the testing room, the experimenter acquainted the subject with the response keys and stimulus display and read the instructions to her. The instructions informed the subject that several blocks of trials would occur and each trial would begin with the onset of a warning signal on the display scope. The warning signal consisted of a $5.08-\mathrm{cm}$ vertical line in the center of the display, flanked on either side by two horizontal $2.54-\mathrm{cm}$ dashes in a single row. The dashes were separated from each other and from the central vertical line by $6.35 \mathrm{~mm}$. Corresponding to the two pairs of dashes were two pairs of response keys on the subject's table. Stimuli consisted of $2.54 \times 2.54 \mathrm{~cm}$ Xs. In double-stimulation blocks, $\mathrm{S}_{1}$ was always an $X$ that appeared above one of the two right-hand dashes, while $S_{2}$ was an $X$ that appeared above one of the left-hand dashes. Thus, both $S_{1}$ and $S_{2}$ involved 1 bit of event uncertainty. In the single-stimulation block, only one $X$ appeared and was presented above one of the two left-hand dashes (i.e., in a position occupied by $\mathrm{S}_{2}$ in double-stimulation blocks). Subjects viewed this display from a distance of $1.5 \mathrm{~m}$, yielding a visual angle for the total display of $4.84 \mathrm{deg}$.

On both single- and double-stimulation trials, the subjects task was to press response keys corresponding to the dashes over which stimulus Xs appeared. The middle and index fingers of each hand were used to respond. In double stimulation there was no explicit requirement that subjects' responses maintain the temporal order of stimulus onsets (i.e., press a right-hand key before responding on a left-hand key to $S_{2}$ ), although this usually occurred. However, there was a special penalty ( -12 points) assessed for responses made before the onset of a corresponding stimulus (i.e., pressing a left-hand key before $S_{2}$ onset or a right-hand key before $S_{1}$ onset). The stimulus display was removed $3.5 \mathrm{sec}$ after the onset of the lefthand stimulus $X$ and replaced by a feedback display that will be described below. When correct responses were made during a trial, corresponding stimulus $\mathrm{Xs}$ were not immediately removed from the screen but remained until the entire stimulus display was cleared from the scope.

Both speed and accuracy of responses were scored and points were awarded according to the payoff matrix in effect. Response speed was judged according to a discrete time criterion that was interactively adjusted during each trial block. Separate criteria were maintained for right- and left-hand responses. The PEST algorithm of Taylor and Creelman (1967) was adapted to this criterion-time adjustment application. The goal of the adjustment process was to select criteria such that each subject could obtain only 75\% accuracy while achieving "fast" responses. This procedure was necessary to insure that payoff conditions would affect each subject's choice of performance strategy. For example, if the criterion for a "fast" response was $3 \mathrm{sec}$, then subjects would not have to choose between fast or accurate performance, since they could always be both "fast" and accurate.

The PEST algorithm differs from the adjustment procedure employed by Knight and Kantowitz (1974) in that criterion adjustments according to the PEST algorithm were of a variable size related to accumulated information on the subject's past responses. This resulted in rapid initial focusing upon desired criteria with increasingly precise adjustments as each trial block progressed. In each block the right- and left-hand criteria were initially set at $350 \mathrm{msec}$ and the eight practice trials provided response histories upon which PEST based its first criteria adjustments.

The feedback display included a left- and right-hand column of information corresponding to each response. For each response this display indicated whether the response was fast or slow, correct or error, and the points earned according to the payoff matrix in effect. Also, at the bottom of the display appeared the total points that the subject had accumulated through the experiment. Subjects were informed that this total was related to monetary reward, with a $\$ 5$ bonus awarded to the high-scoring subject in each group. The feedback display remained on for $4 \mathrm{sec}$ and was followed by a 4-sec intertrial interval before the onset of the next trial's warning signal. Subjects were allowed an ad-lib rest break following each 40 trial block.

\section{RESULTS}

Trials on which null $\left(<1 \%\right.$ for both $R_{1}$ and $\left.R_{2}\right)$ or anticipatory $\left(<1 \%\right.$ for $R_{1},<7 \%$ for $\left.R_{2}\right)$ responses occurred were discarded. To maintain an equal number of observations in each cell of the experimental design, remaining legal trials from the two replications of each double-stimulation trial event in each block (see Design) were averaged; this furnished the basic latency and accuracy data for analysis. (Fortunately, it was never necessary to discard both replications of a particular trial event.) For single-stimulation blocks, two such trial dyads were available for each of the eight singlestimulation trial events. Subsequent latency results are based upon both correct and error responses unless otherwise noted.

\section{First-Response Performance}

First-response accuracy. Figure 2 shows $R_{1}$ accuracy as a function of ISI and SAT (i.e., speed, neutral, or accuracy payoff group). These data were submitted to a four-way analysis of variance, with SAT as a betweensubjects factor and ISI, FP, and Stimulus Pair as withinsubjects factors. Stimulus Pair refers to the four possible $S_{1}$ by $S_{2}$ spatial combinations that could be presented on a trial. These combinations are designated II, IM, MI, and $\mathrm{MM}$ to indicate that correct first and second responses, respectively, required keypresses with index or middle fingers (of the appropriate hand-see Procedure).'

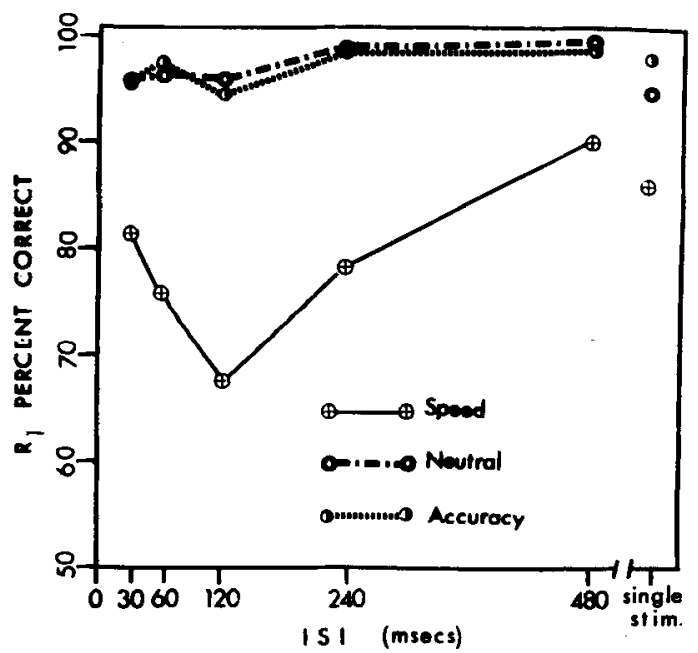

Figure 2. Accuracy of $R_{1}$ as a function of ISI and SAT group. 
The main effect of SAT attained significance $[F(2,15)=8.81, p<.005]$, due primarily to the low accuracy achieved by the speed group (80\%) as compared to the accuracy (98\%) and neutral $(97 \%)$ groups. The latter two groups did not differ significantly $[t(15)=1.01, p>.1]$. While the ISI effect was marginally significant $[F(5,75)=3.13, p<.05]$, Figure 2 shows that this effect was predominantly localized in the speed group. Analysis of variance performed upon only double-stimulation data revealed a significant SAT by ISI interaction $[F(8,60)=2.59, p<.05]$. While the flat ISI functions obtained under accuracy and neutral payoffs are similar to those found by Knight and Kantowitz (1974) under accuracy emphasis, the ISI function for the present speed group differs from that previously observed. While Figure 2 shows that accuracy did decline as ISI was reduced from 480 to $120 \mathrm{msec}$, the increased accuracy at 60 and $30 \mathrm{msec}$ was unexpected. However, though the form of the ISI effect differs from that found previously, the finding that ISI effects upon $R_{1}$ accuracy are most apparent under speed emphasis is consistent with the results of Knight and Kantowitz (1974).

The rise in response accuracy at the two shortest ISIs appeared in the results of all six speed group subjects. Although similar U-shaped performance functions have been infrequently observed (Helson \& Steger, 1962), these involved response latency rather than accuracy. While the present authors are unable to provide a satisfying explanation for this U-shaped function, one clue may lie in the interresponse interval (IRI, the temporal interval separating $R_{1}$ and $R_{2}$ ) data to be presented shortly. As Figure 6 shows, IRI was positive (indicating that the response to $S_{1}$ usually preceded the response to $S_{2}$ ) for all points other than ISI $=30$ and $60 \mathrm{msec}$ for the speed group. The negative IRI values obtained at these two points indicate that responses to $S_{2}$ frequently preceded the response to $S_{1}$. While the connection between reversal of response order and the improved accuracy shown by the speed group subjects at the two shortest ISIs is unclear, a strategy change of some type is suggested by these results.

Finally, $\mathrm{R}_{1}$ accuracy varied among the four possible Stimulus Pairs [MI $=86 \%, \mathrm{MM}=89 \%, \mathrm{II}=96 \%$, and $\mathrm{IM}=95 \%$ correct; $\mathrm{F}(3,45)=4.82, \mathrm{p}<.005]$. NewmanKeuls tests showed that, while the accuracy of Stimulus Pairs MI and MM $\left[\mathrm{q}_{\mathrm{r}}(2,45)=2.38, \mathrm{p}>.05\right]$ and $\mathrm{IM}$ and II $\left[\mathrm{q}_{\mathrm{r}}(2,45)=.05, \mathrm{p}>.1\right]$ did not significantly differ, both IM and II differed from both MI and MM $\left[q_{r} s(45)=2.88, p<.05\right]$. This Stimulus Pair effect can be attributed to an apparent bias toward index-, rather than middle-finger responses: For the 32 experimental trials in each block, the mean number of response pairs observed was $6.3,8.1,10.8$, and 6.7 for MI, MM, II, and IM, where the first and second letters refer to the finger making the first and second responses, respectively. Thus, there were 17.5 mean index-finger

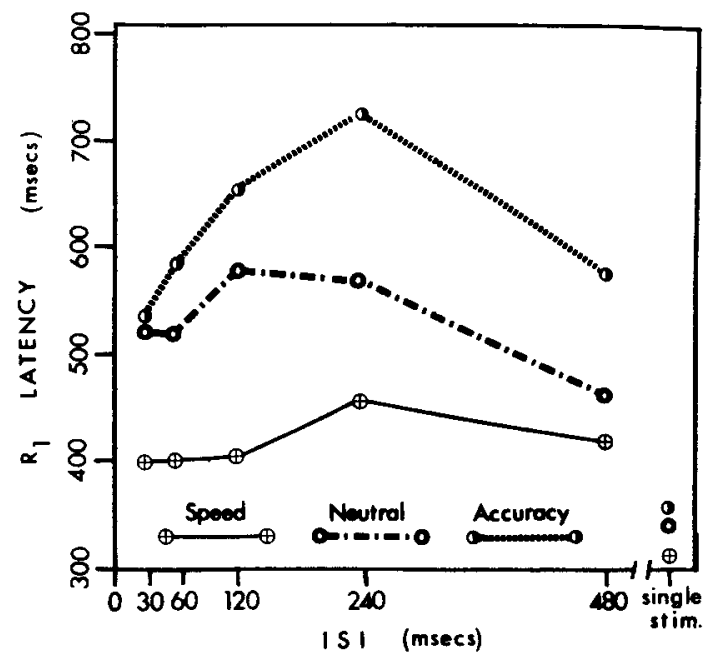

Figure 3. Latency of $R_{1}$ as a function of ISI and SAT group.

responses to $S_{1}(10.8+6.7)$ but only $14.4(6.3+8.1)$ middle-finger $R_{1} s$.

First-response latency. Effects of SAT and ISI upon $\mathrm{RT}_{\mathbf{1}}$ appear in Figure 3. These data were submitted to an analysis identical to that employed for $R_{1}$ accuracy data. The analysis revealed the SAT effect to be statistically significant $[F(2,15)=3.62, p<.05]$. As with the $R_{1}$ accuracy data, a t test failed to discriminate between the double-stimulation $\mathrm{RT}_{1}$ of the accuracy $(619 \mathrm{msec})$ and neutral groups (531 msec) $[\mathrm{t}(15)=1.16$, $\mathrm{MSe}=1,372,354, \mathrm{p}<.1]$.

The effect of ISI upon $\mathrm{RT}_{1}$ which is apparent in Figure 3 was also significant $[F(5,75)=12.62$, $\mathrm{p}<.001]$. However, this ISI effect is not at all reminiscent of the negative exponential shape that usually characterizes the PRP effect. While the form of the present ISI effects are quite different from those found by Knight and Kantowitz (1974), both studies do show a reduction in ISI effect upon $\mathrm{RT}_{1}$ as speed emphasis increases. However, the SAT by ISI interaction failed to achieve statistical significance in the present study $[\mathrm{F}(10,75)=1.23, \mathrm{MSe}=165,310, \mathrm{p}>.05]$. Knight and Kantowitz (1974) found that increased speed emphasis minimized $\mathrm{RT}_{1}$ increases at short ISIs. This form of the present ISI functions suggests that subjects may have intentionally delayed $R_{1}$, perhaps to execute a "grouped" (Welford, 1959) joint $\mathrm{R}_{1}-\mathrm{R}_{2}$ : As ISI increased from 30 to $240 \mathrm{msec}$, subjects may have correspondingly increased the amount of $R_{1}$ delay, perhaps to allow $S_{2}$ processing prior to $R_{1}$ execution. The use of this delaying strategy may have understandably diminished as speed emphasis increased. The decreases in $\mathrm{RT}_{1}$ at $\mathrm{ISI}=480 \mathrm{msec}$ for the accuracy and neutral groups may reflect a reduced use of grouping when the interval between stimuli becomes excessively large (and would therefore require very long $R_{1}$ delays). All single-stimulation $R_{1} s$ were reliably faster than double-stimulation $R_{1} s$ under comparable payoff conditions $[\operatorname{ts}(5)>3.07, \mathrm{p}<.05]$. 
Finally, as with $R_{1}$ accuracy, $R T_{1}$ was found to depend upon Stimulus Pair, with $\mathrm{RT}_{1}=534,487,451$, and $494 \mathrm{msec}$ for MI, MM, II, and IM pairs, respectively $[F(3,45)=11.58, p<.001]$. In agreement with the $R_{1}$ accuracy data, a contrast showed that responses made with the right-hand index finger were reliably faster than responses made with the right-hand middle finger $[t(45)=3.45, p<.05]$. No other effects upon $R_{1}$ achieved statistical significance.

First-response information transmission rate. Since performance in the present experiment depended upon both speed and accuracy of response, assessment based upon a dependent variable that accounts for both performance dimensions is mandated. The present authors selected information transmission rate (transmitted information/ $\overline{\mathrm{RT}}$ ), $\mathrm{H}_{\mathrm{t}} / \mathrm{sec}$, as the measure, since it has previously been shown to reflect both speed and accuracy decrements associated with short ISIs (Knight \& Kantowitz, 1974). A disadvantage of this metric is its sensitivity to response bias (Moray \& Fitter, 1973). Such bias reduces $\mathrm{H}_{t} / \mathrm{sec}$ below levels attainable in the absence of bias. Since the Stimulus Pair effects found for both $R_{1}$ accuracy and latency suggest the presence of response bias, extra caution must be exercized in interpreting the $\mathrm{H}_{\mathrm{t}} / \mathrm{sec}$ results.

For each block of 32 experimental trials, legal $R_{1} s$ were entered into a 2 by 2 (stimulus by response) transmitted information matrix and mean $\overline{\mathrm{RT}}_{1}$ for the block was determined. Dividing transmitted information by $\mathrm{RT}_{1}$ yielded the datum of interest in each block. Each subject thus furnished six observations for analysis, one for each ISI.

Information transmission rates for $R_{1}$ are presented in Table 1 as a function of SAT and ISI. A two-way analysis of variance (SAT by ISI) ${ }^{2}$ upon Table 1 data revealed significant effects of $\operatorname{SAT}[F(2,10)=7.18$, $\mathrm{p}<.01]$ and ISI $[\mathrm{F}(4,20)=14.30, \mathrm{p}<.001]$. The SAT by ISI interaction, however, failed to attain significance $[F(8,40)=1.37, \mathrm{MSe}=.2449, \mathrm{p}>.05]$. Also, a $t$ test again failed to discriminate performance of the neutral group from that of the accuracy group $[t(10)=1.35, p>.1]$. Both of these groups, however, were superior to the speed group $[\operatorname{ts}(10)>2.67$, $p<.05]$. Performance under speed emphasis has consistently been found to produce lower $\mathrm{H}_{t} / \mathrm{sec}$ when compared to an accuracy emphasis (Fitts, 1966; Knight \& Kantowitz, 1974): Subjects fail to compensate

Table 1

First-Response Information Transmission Rates

\begin{tabular}{lrrrrrrr}
\hline & \multicolumn{7}{c}{ ISI (msec) } \\
\cline { 2 - 7 } \multicolumn{1}{c}{ Group } & \multicolumn{1}{c}{ 30 } & 60 & 120 & 240 & 480 & SS & $\overline{\mathrm{X}}$ \\
\hline Speed & 1.34 & .88 & .35 & .78 & 1.94 & 1.79 & 1.18 \\
Neutral & 1.64 & 1.75 & 1.58 & 1.76 & 2.40 & 2.29 & 1.90 \\
Accuracy & 1.61 & 1.61 & 1.32 & 1.42 & 1.90 & 2.58 & 1.74 \\
$\overline{\mathrm{X}}$ & 1.53 & 1.41 & 1.08 & 1.32 & 2.08 & 2.22 & 1.61 \\
\hline
\end{tabular}

Note-Table entries are in bits/second, $M S e=.245, S S=$ single stimulation.

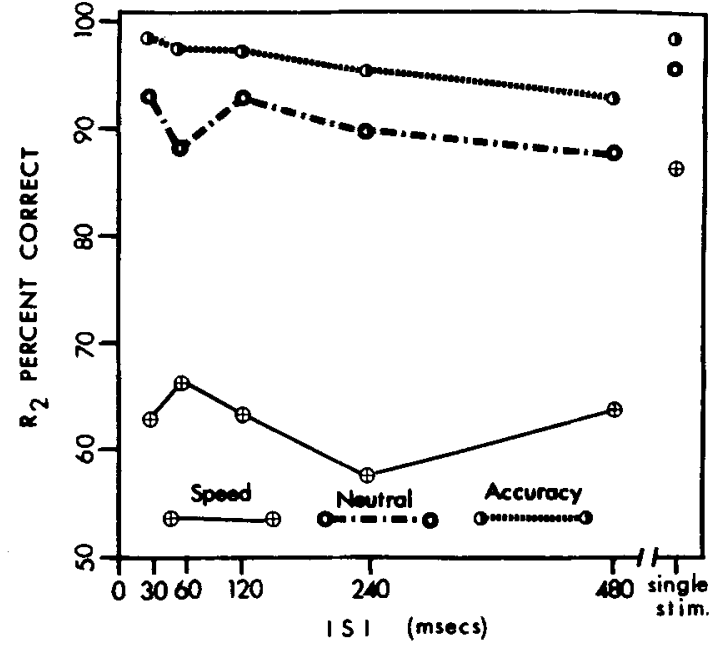

Figure 4. Accuracy of $R_{2}$ as a function of ISI and SAT group.

accuracy losses with sufficient gains in response speed. While $\mathrm{H}_{\mathrm{t}} / \mathrm{sec}$ generally decreased as ISI was reduced from 480 to $120 \mathrm{msec}$, there was an unexpected rise in performance at very short ISIs. For the speed group it is clear that this effect is related to the increase in accuracy at short ISIs rather than to decreased latency. Such a sharp break in the ISI function suggests a fundamental processing change at roughly $I S I=120 \mathrm{msec}$.

\section{Second-Response Performance}

Second-response accuracy. Second-response accuracy is shown in Figure 4 as a function of SAT and ISI. These data were submitted to an analysis of variance similar to that employed for $R_{1}$ data. Note that the singlestimulation points in Figure 4 are the same as those in Figure 3 since the same single-stimulation responses provided baselines for both $R_{1}$ and $R_{2}$. The main effect of SAT proved significant $[F(2,15)=28.64$, $\mathrm{p}<.001]$, as did the main effect of ISI $[F(5,75)=3.70$, $p<.01]$. However, this ISI effect is attributable to the difference between single- and double-stimulation performance. An additional analysis of variance on only double-stimulation data revealed no ISI effect $[F(4,60)<1]$. Furthermore, Dunnett tests revealed that only under speed emphasis did double stimulation differ significantly from single stimulation $[d(15)=5.02, p<.05]$, although the SAT by ISI interaction did not attain statistical significance $[\mathrm{F}(10,75)=1.58, \mathrm{MSe}=.1622, \mathrm{p}>.1]$. For double stimulation only, the speed group accuracy $(63 \%$ correct) was significantly above chance $(50 \%)$ by a onesample $t$ test $[t(15)=3.56, p<.01]$. Also, while the speed group was reliably less accurate than either the neutral or accuracy groups [Newman-Keuls $\left.\mathrm{q}_{\mathrm{r}} \mathrm{s}(15)>8.18, \mathrm{p}<.001\right]$, these latter two groups were not distinguished $\left[\mathrm{q}_{\mathrm{r}}(2,15)=1.89, \mathrm{p}>.1\right]$. Thus, again there is little evidence for an intermediate SAT strategy in the present experiment.

More importantly, however, Figure 4 shows that the double-stimulation performance decrement appears 
in the form of reduced accuracy when speed is emphasized, since $R_{2}$ accuracy for the speed group is well below its single-stimulation control baseline. This is consistent with conclusions drawn in the earlier Knight and Kantowitz (1974) paper. However, the present results do not reveal increasing accuracy decrements as ISI is reduced, analogous to the increasing $R_{2}$ delays that characterize the PRP effect. Thus, these $R_{2}$ results contrast with the $R_{1}$ effects found by Knight and Kantowitz (1974), who observed increasing $R_{1}$ inaccuracy with decreasing ISI.

As with $R_{1}$ accuracy, $R_{2}$ accuracy was found to depend upon Stimulus Pair $[\mathrm{MI}=83 \%, \quad \mathrm{II}=92 \%$, $\mathrm{MM}=85 \%$, and $\mathrm{IM}=76 \%$ correct, $\mathrm{F}(3,45)=8.47$, $\mathrm{p}<.001]$. Unlike $R_{1}$, however, for which index-finger responses were basically faster and more accurate than middle-finger responses, $R_{2}$ accuracy depended upon particular combinations of both $S_{1}$ and $S_{2}$. While Stimulus Pair II produced highest accuracy in all three payoff groups, this advantage was greatly diminished under accuracy emphasis, resulting in a significant SAT by Stimulus Pair interaction $[F(6,45)=3.01$, $p<.05]$. No other significant effects upon $R_{2}$ accuracy were found.

Second-response latency. Figure 5 presents $R_{2}$ as a function of SAT and ISI. While the main effect of SAT was significant $[F(2,15)=10.64, p<.005]$, once again a $t$ test failed to discriminate between the neutral and accuracy groups $[\mathrm{t}(15)=1.89$, $\mathrm{MSe}=597,950, \mathrm{p}>.1 \mathrm{]}$, thus cornplementing a similar result for $R_{2}$ accuracy. Both neutral and accuracy groups differed from the speed group by a NewmanKeuls test $\left[\mathrm{q}_{\mathrm{r}}(15)>3.85, \mathrm{p}<.05\right]$.

The main effect of ISI also proved statistically significant $[F(5,75)=10.15, p<.001]$. The doublestimulation $\mathrm{RT}_{2} \mathrm{~S}$ in Figure 5 consistently increase as ISI is reduced from 480 to $30 \mathrm{msec}$ in a manner characteristic of the PRP effect. Although the SAT by ISI interaction was not statistically reliable

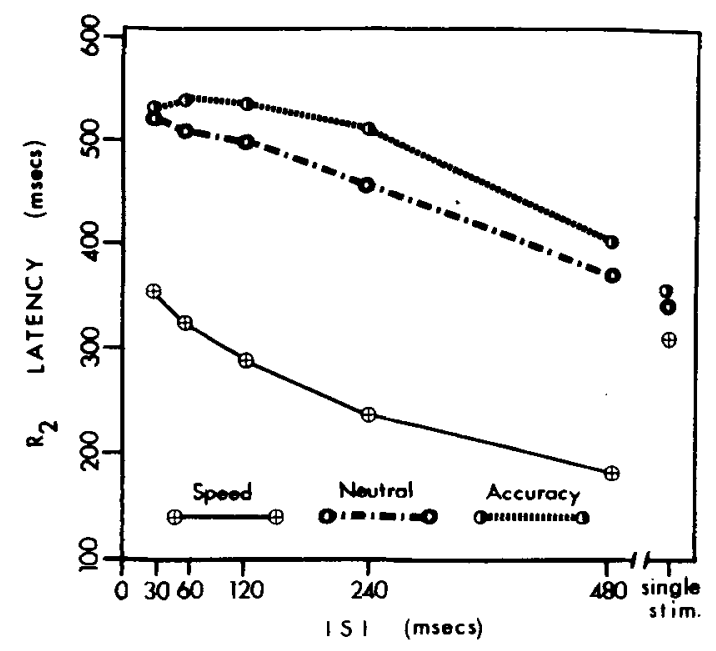

Figure 5. Latency of $R_{2}$ as a function of ISI and SAT group.
$[F(10,75)=1.60, \mathrm{MSe}=113,041, \mathrm{p}>.1]$, comparisons between single- and double-stimulation data points in Figure 5 suggest that quite different processes are mediating ISI effects in the speed group as compared to the neutral and accuracy groups. Figure 5 dramatically points out the necessity for proper single-stimulation baselines in evaluating double-stimulation performance (Kantowitz, 1974a). While the $\mathrm{RT}_{2}$ functions for the neutral and accuracy groups evince a PRP effect with increasing performance decrement as ISI is reduced, the $\mathrm{RT}_{2}$ function for the speed group is better interpreted as showing decreasing facilitation as ISI is reduced. Indeed, under speed emphasis $\mathrm{RT}_{2}$ shows no double-stimulation decrement at short ISIs relative to a single-stimulation control baseline and thus does not reflect a PRP effect. Examination of both Figures 4 and 5 does make clear, however, that the performance decrement associated with short ISIs shows up in the form of increased $R_{2}$ latency (i.e., a PRP effect), rather than inaccuracy, when performance accuracy is emphasized. Thus, the $R_{2}$ data obtained in the present study are quite compatible with the $R_{1}$ data and conclusions drawn therefrom by Knight and Kantowitz (1974).

While the decreasing $\mathrm{RT}_{2}$ functions obtained under neutral and accuracy payoffs reflected a PRP effect, this was not true of the $\mathrm{RT}_{2}$ function for the speed group. Under speed emphasis the decreasing $\mathrm{RT}_{2}$ function suggests the use of a response strategy in which a preselected $R_{2}$ (Yellott, 1971) is executed at a preset time after $R_{1}$ with minimal $S_{2}$ analysis per se. Such an explanation is also consistent with anticipatory response data. For the speed group there were 71 anticipatory $R_{2} s$. Of these, 19 occurred at ISI $=240 \mathrm{msec}$ and 52 occurred at ISI $=480 \mathrm{msec}$, suggesting increased error in subjects' temporal estimations. No anticipations were found in the accuracy group and only one (at ISI $=240 \mathrm{msec}$ ) was found in the neutral group.

Second-response latency was also affected by Stimulus Pair, with $\mathrm{RT}_{2}=423,401,366$, and $426 \mathrm{msec}$ for MI, MM, II, and IM, respectively $[\mathrm{F}(3,45)=11.71$, $\mathrm{p}<.001]$. Newman-Keuls tests showed that II produced faster $R_{2} s$ than any other Stimulus Pair $\left[q_{r} s(45)>4.36\right.$, $\mathrm{p}<.01]$, while no significant differences among the other Stimulus Pairs appeared $\left[\mathrm{q}_{\mathrm{r}} \mathrm{s}(45)<3.16, \mathrm{p}>.05\right]$. However, for the longest double-stimulation ISI (480 msec), II and MI produced equally fast $R_{2} \mathrm{~s}$ (306 and $307 \mathrm{msec}$, respectively), indicating a reduced influence of $S_{1} \cdot R_{1}$ upon $R_{2}$. This change in MI performance resulted in a significant ISI by Stimulus Pair interaction $[F(15,225)=2.36, p<.01]$. No other effects proved significant.

Second-response information transmission rates. Information transmission rates for $\mathbf{R}_{\mathbf{2}}$ were computed in a manner identical to that employed for $R_{1}$. The resulting data are displayed in Table 2 as a function of SAT and ISI. A two-way analysis of variance, identical 
Table 2

Second-Response Information Transmission Rates

\begin{tabular}{lrrrrrrr}
\hline & \multicolumn{7}{c}{ ISI (msec) } \\
\cline { 2 - 7 } \multicolumn{1}{c}{ Group } & \multicolumn{1}{c}{30} & 60 & 120 & 240 & 480 & SS & $\overline{\mathrm{X}}$ \\
\hline Speed & .42 & .35 & .39 & .71 & .65 & 1.79 & .72 \\
Neutral & 1.40 & 1.10 & 1.36 & 1.27 & 1.43 & 2.29 & 1.48 \\
Accuracy & 1.80 & 1.65 & 1.71 & 1.67 & 1.71 & 2.58 & 1.85 \\
$\overline{\mathrm{X}}$ & 1.21 & 1.03 & 1.15 & 1.22 & 1.26 & 2.22 & 1.35 \\
\hline
\end{tabular}

Note-Table entries are in bits/second, $M S e=.325, S S=$ single stimulation.

to that employed for $R_{1} H_{t} / s e c$ data, revealed a significant effect of SAT $[F(2,10)=14.02, p<.001]$. However, the ordering of the three experimental groups differed from that observed in the $R_{1}$ analysis, for which the neutral group produced the best performance. For $\mathbf{R}_{\mathbf{2}}$, the accuracy group produced the best performance, while the speed group produced the lowest $\mathrm{H}_{\mathrm{t}} /$ sec. Newman-Keuls tests revealed significantly lower performance in the speed group than in the accuracy and neutral groups $\left[\mathrm{q}_{\mathrm{r}} \mathrm{s}(10)>5.36, \mathrm{p}<.01\right]$, although the latter two groups did not differ significantly $\left[\mathrm{q}_{\mathrm{r}}(2,10)=2.70, \mathrm{p}>.05\right]$.

In addition, a significant effect of ISI was found $[F(5,25)=14.18, p<.001]$. However, this was clearly due to the contrast between single- and doublestimulation performance. A second analysis of only double-stimulation data did not indicate a significant ISI effect $[F(4,20)<1]$. Thus, these data contrast with the $R_{1}$ data obtained in the present experiment, and by Knight and Kantowitz (1974) which showed progressive $\mathrm{R}_{1} \mathrm{H}_{t} / \mathrm{sec}$ decrements as ISI was reduced. There was no indication of an SAT by ISI interaction in the present $\mathrm{R}_{2} \mathrm{H}_{\mathrm{t}} / \mathrm{sec}$ data.

\section{Joint First- and Second-Response Performance}

Interresponse interval (IRI). To further examine possible response grouping and to determine if joint performance was characterized by a fixed minimum IRI (Keele, 1973), the interval separating $R_{1}$ and $R_{2}$ on each trial was determined. This data is presented in Figure 6 as a function of ISI and SAT. An analysis of variance performed on the data of Figure 6 revealed that only the main effect of ISI $[F(4,60)=55.88$, $p<.001]$ attained statistical significance. This ISI effect can best be interpreted in two parts. For ISI $<240 \mathrm{msec}(<120 \mathrm{msec}$ for neutral), IRI is constant and almost zero. This is indicative of a high degree of response grouping. Since $\mathbf{R}_{2}$ delay relative to singlestimulation baselines was greatest at these short ISIs for the neutral and accuracy groups, these results do not support the view that the PRP effect is due to an irreducible minimum IRI. For longer ISIs, however, subjects abandoned response grouping and opted for more independent responses. Thus, ISI appears to be related to limits of response grouping as well as stimulus grouping (Welford, 1959). While the temporal point at which the accuracy and speed groups abandoned response grouping did not appear to differ, examination of $\mathrm{RT}_{1}$ and $\mathrm{RT}_{2}$ data showed that these groups did differ in the way they produced grouped responses. For the accuracy group, $R_{1}$ was delayed to bring it closer to $R_{2}$, while for the speed group, $R_{2}$ was speeded through response timing to bring it closer to $R_{1}$. Thus, these data reveal two distinct processes for response grouping not previously distinguished. The constant IRI produced by the neutral group at the three shortest ISIs cannot unambiguously be ascribed to only one of the response grouping mechanisms just described but probably reflects a combination of some $R_{1}$ delay and increased $R_{2}$ speed as PRP decrements are reduced.

Conditional response analysis. Since there were two possible $R_{1}$ s and two possible $R_{2} s$, any of four possible $R_{1}$ by $R_{2}$ response pairs could occur on each doublestimulation trial. To illuminate further responsegrouping and response-bias effects, the total number of each type of response pair at each ISI was tabulated for each of the three experimental groups. These totals are shown in Table 3. A three-way (SAT by ISI by Response Pair) analysis of variance was performed on the data of Table 3. The main effect of Response Pair was significant $[F(3,45)=10.97, p<.001]$. An orthogonal contrast between same-finger response pairs (MM and II) and different-finger response pairs (IM and MI) revealed a greater occurrence of the former $[t(45)=4.83, p<.01]$. While this preference appeared in all three experimental groups to some degree, it is clear that the domination of this response pattern is strongest in the speed group $[F(6,45)=4.45, p<.005]$, with II response pairs being most popular. Since samefinger response pairs do not correspond to a simple stimulus pattern (e.g., both stimuli on left), these response-pair effects emphasize the importance of

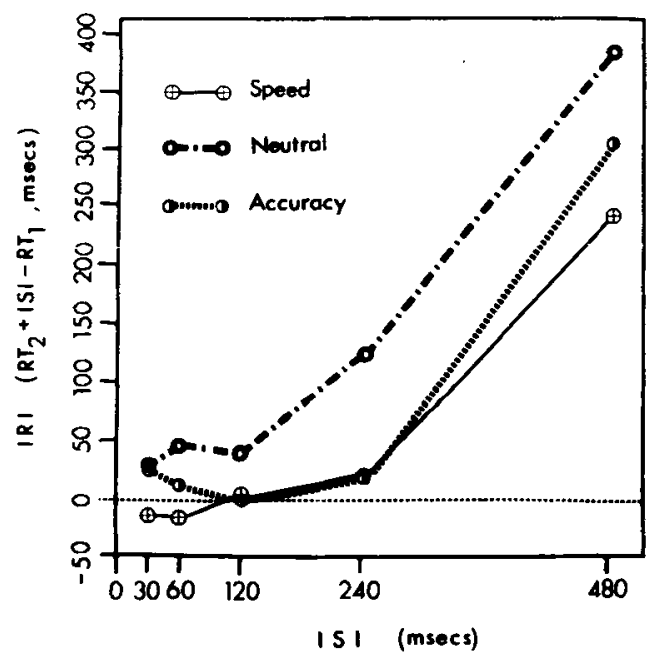

Figure 6. Interresponse interval $\left(R_{2}+I S I-R T_{1}\right)$ as a function of ISI and SAT group. 
Table 3

Conditional Response Analysis

\begin{tabular}{|c|c|c|c|c|c|}
\hline Group & ISI (msec) & MI & MM & II & IM \\
\hline Speed & $\begin{array}{r}30 \\
60 \\
120 \\
240 \\
480 \\
\text { SS } \\
\Sigma\end{array}$ & $\begin{array}{r}27 \\
20 \\
24 \\
31 \\
32 \\
96 \\
230\end{array}$ & $\begin{array}{r}48 \\
46 \\
44 \\
48 \\
46 \\
93 \\
325\end{array}$ & $\begin{array}{r}97 \\
87 \\
73 \\
89 \\
88 \\
2 \\
436\end{array}$ & $\begin{array}{r}20 \\
39 \\
51 \\
24 \\
26 \\
1 \\
157\end{array}$ \\
\hline Neutral & $\begin{array}{r}30 \\
60 \\
120 \\
240 \\
480 \\
\text { SS } \\
\Sigma\end{array}$ & $\begin{array}{r}43 \\
36 \\
37 \\
45 \\
44 \\
95 \\
300\end{array}$ & $\begin{array}{r}52 \\
54 \\
54 \\
50 \\
52 \\
97 \\
359\end{array}$ & $\begin{array}{r}52 \\
64 \\
55 \\
59 \\
48 \\
0 \\
278\end{array}$ & $\begin{array}{r}44 \\
37 \\
46 \\
38 \\
48 \\
0 \\
213\end{array}$ \\
\hline Accuracy & $\begin{array}{r}30 \\
60 \\
120 \\
240 \\
480 \\
S S \\
\Sigma\end{array}$ & $\begin{array}{r}43 \\
47 \\
42 \\
51 \\
47 \\
94 \\
324\end{array}$ & $\begin{array}{r}49 \\
47 \\
51 \\
43 \\
48 \\
97 \\
335\end{array}$ & $\begin{array}{r}56 \\
50 \\
54 \\
50 \\
49 \\
1 \\
260\end{array}$ & $\begin{array}{r}44 \\
48 \\
42 \\
48 \\
48 \\
0 \\
230\end{array}$ \\
\hline
\end{tabular}

Note-Table entires are observed totals of each type of response pair. The first letter $(I=$ index, $M=$ middle) refers to the finger making $R_{1}$ and the second letter refers to the finger making $R_{2} . S S=$ single stimulation.

response- rather than stimulus-processing mechanisms, especially as speed emphasis increases. Of additional interest for the speed group is the finding that the same-finger response pattern does not diminish even at the longest ISI, where the IRI data revealed considerable separation of responses. A speculation might be that subjects made only a single response selection as a strategy to minimize double-stimulation performance decrements. These data also suggest that, as speed stress increases, response predispositions and biases play an increasingly important role in performance. No other statistically significant effects were observed in the Table 3 data.

Latency of correct vs incorrect responses. A number of competing models of the speed-accuracy tradeoff make differing predictions concerning the relative latencies of correct and error responses. Fast-guess models typically (though not necessarily) suggest faster error responses (Yellott, 1971); deadline models (Swensson, Note 2) and response-conflict models (Kantowitz, 1969; Knight \& Kantowitz, 1974) suggest slower errors; random-walk models (Fitts, 1966; Link, Note 3) suggest equal latencies for correct and error responses. Knight and Kantowitz (1974), while finding slower incorrect first responses in double stimulation, found the opposite in single stimulation where errors were faster than correct responses. Table 4 shows mean correct- and error-response latencies for the various conditions in the present experiment. The $R_{1}$ data replicate the general finding of Knight and Kantowitz (1974): Slower error responses may appear in double stimulation but not in single stimulation. This result appears to depend upon SAT, although the low number of incorrect responses generated by the neutral and accuracy groups precludes firm conclusions.

\section{DISCUSSION}

The principal conclusion to be drawn from these data is that $R_{2}$ double-stimulation performance and, in particular, the PRP effect are sensitive to task SAT demands in much the same manner as found for $R_{1}$ by Knight and Kantowitz (1974). Examination of $\mathrm{RT}_{2}$ data revealed a PRP effect only under accuracy and neutral payoff conditions. Analogously, Knight and Kantowitz (1974) found $\mathrm{RT}_{1}$ elevations at short ISIs only under accuracy emphasis. This similarity of effects provides some support for a unitary mechanism (Kantowitz, 1974b) underlying both $R_{1}$ and $R_{2}$ effects in double stimulation.

While the $\mathrm{RT}_{2}$ ISI function produced by the speed group in the present study posessed a shape that is typical of the PRP effect, comparison with a singlestimulation control RT clearly showed that this function could not be described as a true PRP effect. Under speed stress $\mathrm{RT}_{2}$ at the shortest $(30 \mathrm{msec}$ ) did not significantly exceed the single-stimulation baseline and, as ISI increased, double-stimulation $\mathrm{RT}_{2}$ dropped far below this value. Thus, this $\mathrm{RT}_{2}$ function is better interpreted as showing decreasing double-stimulation facilitation, rather than increasing decrement (a characteristic of the PRP effect), as ISI decreases.

Under speed stress subjects apparently adopted a

Table 4

Comparison of Mean Correct- and Error-Response Latencies

\begin{tabular}{|c|c|c|c|c|c|c|}
\hline \multirow[b]{2}{*}{ Group } & \multicolumn{4}{|c|}{$\begin{array}{r}\text { First Response } \\
\text { Double Stimulation }\end{array}$} & \multicolumn{2}{|c|}{$\begin{array}{l}\text { Second Response } \\
\text { Double Stimulation }\end{array}$} \\
\hline & Error & Correct & Error & Correct & Error & Correct \\
\hline Speed & $\begin{array}{c}293 \\
(26 ; 65)\end{array}$ & $\begin{array}{c}316 \\
(166 ; 79)\end{array}$ & $\begin{array}{c}360 \\
(202 ; 167)\end{array}$ & $\begin{array}{c}433^{*} \\
(758 ; 183)\end{array}$ & $\begin{array}{c}239 \\
(324 ; 122)\end{array}$ & $\begin{array}{c}336^{*} \\
(565 ; 163)\end{array}$ \\
\hline Neutral & $\begin{array}{l}279 \\
(9 ; 69)\end{array}$ & $\begin{array}{c}344 * \\
(183 ; 70)\end{array}$ & $\begin{array}{c}595 \\
(20 ; 276)\end{array}$ & $\begin{array}{c}526 \\
(940 ; 214)\end{array}$ & $\begin{array}{c}346 \\
(35 ; 90)\end{array}$ & $\begin{array}{c}478^{*} \\
(924 ; 186)\end{array}$ \\
\hline Accuracy & $\begin{array}{l}272 \\
(3 ; 22) \\
\end{array}$ & $\begin{array}{c}360^{*} \\
(189 ; 87)\end{array}$ & $\begin{array}{c}702 \\
(27 ; 494)\end{array}$ & $\begin{array}{c}607 \\
(936 ; 257)\end{array}$ & $\begin{array}{c}336 \\
(97 ; 135)\end{array}$ & $\begin{array}{c}512^{*} \\
(864 ; 164)\end{array}$ \\
\hline
\end{tabular}

Note-Table entries are mean $R T s$ in milliseconds. Numbers in parentheses are ( $N$; standard deviation).

*Correct $R T$ - error RT significant at $p<.01$. 
timed-response mode with minimal $S_{2}-R_{2}$ processing (Kornblum, 1973). Utilizing this response mode, subjects could execute $R_{2}$ at a preset interval following $R_{1}$ : As shown by the IRI data, this interval was often zero. This processing mode may have been partially induced by the blocked-ISI procedure utilized in the present study. Similarly, Knight and Kantowitz (1974) found a timed-response mode to predominate under speed emphasis but attributed this to the lack of conditional $R_{2}$ event uncertainty in that study. It is somewhat more surprising that subjects adopted a similar response mode under conditions of $S_{2}-R_{2}$ uncertainty, especially since some value, although relatively small, was placed upon response accuracy even under speed emphasis and anticipatory responses were penalized.

The timed-response mode which appeared under speed stress is similar to fast-guess responding as described by Yellott (1971) and by Ollman (1966). However, in this fast-guess response mode a preselected response is triggered by the detection of stimulus onset, rather than termination of a timing process initiated by a preceding stimulus or response. Another recent variant of the fast-guess mechanism (Swensson, Note 2) is based upon expiration of a temporal deadline; this timing process is not initiated by a preceding stimulus or response, but rather by the onset of the stimulus to which the subject is required to respond. A question that is not clearly answered by the present data is whether the timing process apparently used in the present experiment was initiated by $S_{1}$ or by $R_{1}$. This problem might be attacked by intermixing trials which present an $S_{1}$ not mapped to an overt response among more traditional dual-response trials. The most important general conclusion to be drawn here, however, is that SAT demands may be a strong influence, not only on quantitative aspects of $S_{2} \cdot R_{2}$ processing, but also upon the qualitative nature of a subject's processing strategy.

While it is possible that some of the $\mathrm{RT}_{2}$ decline with increasing ISI under neutral and accuracy payoffs was also due to contributions of timed responses, this would not seem to account for the entire effect for two reasons. First, there was an essentially complete lack of anticipatory $R_{2} s$ under neutral and accuracy payoffs. At least some anticipations would be expected at the longest ISI under a timed-response mode. Second, $\mathrm{RT}_{2}$, even at the longest ISI $(480 \mathrm{msec}$, where timed responses would be potentially most useful), was above the single-stimulation baseline RT. Since timed responses were not possible under single stimulation, the use of this response mode would be expected to produce $\mathrm{RT}_{2} \mathrm{~s}$ that were below the single-stimulation RT (as found with the speed group).

In agreement with expectations based upon the $R_{1}$ data of Knight and Kantowitz (1974), the present $\mathbf{R}_{\mathbf{2}}$ data showed that under speed stress the doublestimulation performance decrement appeared in the form of reduced accuracy, rather than speed. For the neutral and accuracy groups, $R_{2}$ accuracy showed minimal double-stimulation decrement. Thus, as with $R_{1}$, subjects were able to select the form in which double-stimulation limitations are manifest so as to minimize their impact upon task requirements.

In contrast to the $R_{1}$ accuracy results obtained for their speed-emphasis group by Knight and Kantowitz (1974), the present $R_{2}$ results do not show increasing double-stimulation accuracy with lengthening ISI. Rather, there is a constant double-stimulation accuracy decrement for the present speed group compared to its single-stimulation baseline. This contrast between the present and earlier studies may stem from at least two sources. First, it is clear that the use of a timed-response strategy in double stimulation could lead to a constant and drastic reduction in accuracy at all ISIs. Thus, subjects may have used timed responses on a fixed proportion of trials regardless of ISI.

A second possibility may lie in the fact that the previous study involved overlap of $S_{1}$ and $S_{2}$ as well as $R_{1}$ and $R_{2}$ sets (i.e., $S_{1}$ and $S_{2}$ were selected from the same set of possibilities; this was also true for $R_{1}$ and $R_{2}$ ), while in the present study first and second stimulus and response sets were disjoint. Herman (1969) hypothesized and experimentally demonstrated minimal $\mathrm{RT}_{1}$ double-stimulation decrements with such disjoint sets. Herman and Kantowitz (1970) considered such an arrangement to be neutral in that neither $\mathrm{RT}_{1}$ nor $\mathrm{RT}_{2}$ would necessarily show detrimental effects of decreasing ISI. Thus, the present $R_{2}$ accuracy data might be explained by a combination of timed responses (thus accounting for the general double-stimulation accuracy decrement) and the use of disjoint stimulus and response sets (thus accounting for the lack of ISI effect upon $R_{2}$ accuracy).

The above explanation for the lack of ISI effects upon $R_{2}$ accuracy (i.e., there was no ISI effect because first and second stimulus and response sets were disjoint) seems to imply that no PRP delays should be found in the $\mathrm{RT}_{2}$ data. However, in elaborating the responseconflict model, Kantowitz (1974b) has pointed out that disjoint first and second stimulus and response sets may yield $\mathrm{RT}_{2}$ delays (e.g., Kantowitz \& Sanders, 1972); indeed, a PRP effect was evident for both the neutral and accuracy groups. The resolution of this apparent difficulty may lie in Herman's (1969) distinction between directly aroused response conflict and mediated arousal. Direct conflict arousal results from the presentation of $S_{1}$, which elicits its associated response tendency. It is primarily the correct response tendency associated with $S_{1}$ that thus interferes with the selection of the correct response to $S_{2}$. This rapid process accounts for performance decrements at very short ISIs. At longer ISIs, mediated arousal of competing response tendencies occurs through generalization among similar response tendencies. This second, slower process is intended to account for $\mathrm{RT}_{2}$ delays at longer ISIs. Since 
the process of mediated arousal is slower than direct arousal, it might be expected to appear only in the $\mathrm{RT}_{2}$ data of the accuracy and neutral groups: In the speed group $R_{2}$ may have been executed before this source of conflict had developed. In the present experiment response tendencies may have become linked by generalization through temporal proximity.

While analysis of $R_{2}$ performance by itself demonstrated effects of SAT demands, it is clear from joint $R_{1}-R_{2}$ performance that overall double-stimulation strategies were selected upon the basis of the task payoff structure. Thus, accuracy and neutral groups, while exhibiting PRP effects also showed evidence for response grouping, a strategy in which $R_{1}$ may be delayed until completion of $\mathrm{S}_{2}$ processing when a single molar response is executed to both $S_{1}$ and $S_{2}$. Welford (1959) suggested that subjects might utilize stimulus grouping at sufficiently short ISIs and that this might minimize the PRP effect. This grouping concept was extended to response grouping by Borger (1963) under the assumption that either $R_{1}$ execution or the feedback stimuli resulting therefrom might interfere with $S_{2}$ processing. Therefore, delaying $R_{1}$ until completion of $\mathrm{S}_{2}$ processing might prove beneficial. The strong evidence for response grouping in the present experiment comes from the $\mathrm{RT}_{1}$ data of the neutral and, especially, the accuracy groups which show increasing $\mathrm{RT}_{1}$ with increasing ISI. Since the apparent grouping extends beyond the limits of stimulus grouping proposed by Welford, it appears that subjects used response grouping to minimize the PRP effect. The use of response grouping was strongest with the accuracy payoff, which is reasonable since the cost of this strategy was minimized under the accuracy payoff structure. The use of response grouping may account for the rather shallow slopes of the $\mathrm{RT}_{2}$ ISI functions.

It is also clear that response grouping utilized by the present accuracy and neutral groups substantially accounts for the distinct qualitative differences between the $\mathrm{RT}_{1}$ functions obtained under accuracy emphasis by Knight and Kantowitz (1974) and those of the present study. In the earlier paper $\mathrm{RT}_{1}$ decreased consistently with increasing ISI under accuracy but not under speed emphasis. While response timing was found for $R_{2}$ under speed stress, there was no indication in the earlier study of the second grouping mechanism (delay of $R_{1}$ ) under accuracy emphasis. However, in this earlier study $R_{2}$ was completely constrained by $R_{1}$ and, optimally, required no decisions on the part of the subject other than the timing of $R_{2}$ execution. It also seems probable that the attractiveness of the grouping strategy is related to overlap of stimulus and/or response sets. Thus, Kantowitz (1974b) also found decreasing or constant $\mathrm{RT}_{1}$ with increasing ISI under conditions of $R_{2}$ event uncertainty coupled with overlap of both stimulus and response sets.

Consideration of joint $R_{1}-R_{2}$ performance also reveals a point noted by the anonymous reviewer of this manuscript: Overall, $R_{1}$ performance was more strongly influenced by ISI than was $R_{2}$ performance. This is the reverse of usual findings in PRP studies (Kantowitz, 1974b) and is most likely attributable to the response-grouping processes described above. In studies showing smaller $\mathrm{RT}_{1}$ than $\mathrm{RT}_{2}$ effects, $\mathrm{RT}_{1}$ declines with increasing ISI. The discrepency between the present study and previous studies regarding the ordering of the magnitudes of $R_{1}$ and $R_{2}$ effects probably reflects the relative influences of two different mechanisms for $R_{1}$ effects: Response grouping, evidenced by increasing $R T_{1}$ with increasing ISI, may yield larger $R_{1}$ than $R_{2}$ effects, while processes producing decreasing $\mathrm{RT}_{1}$ with increasing ISI may frequently yield larger $R_{2}$ effects. Conflict during response selection has been supported as a likely candidate for this second process (Herman \& Kantowitz, 1970; Knight \& Kantowitz, 1974). An important question which is not answered by present or previous results is the conditions under which response grouping, rather than response (selection) conflict, predominates in double stimulation. It is likely that this would depend upon specific task payoff systems and other important double-stimulation variables, particularly the range of ISIs employed, the sizes of stimulus and response sets, and interrelations between first and second stimulusresponse sets.

The present grouping results have methodological implications for further studies of this processing strategy. It would appear that manipulation of SAT demands might provide a way of controlling and experimentally varying subjects' use of response grouping. Previously such manipulation was available only through the use of explicit differential instructions. The use of SAT payoff contingencies may afford a means of obtaining a more continuous variation in the use of response grouping.

The $\mathrm{RT}_{2}$ data have negative implications for at least two other competing explanations of the PRP effect. First, it is difficult to ascribe the PRP effect to decreased stimulus discriminability at short ISIs, since $S_{1}$ and $S_{2}$ were selected from entirely different subsets. Also, explanations based upon eye movement are not viable, since both $S_{1}$ and $S_{2}$ occurred within the angle of foveal vision. Second, Keele (1973) has proposed a model of the PRP effect based upon a minimum time between successive response initiations. In the present case, examination of IRI data revealed interresponse times near zero even for the neutral and accuracy groups which clearly showed a PRP effect. To the extent that IRI accurately reflects the time between successive central response initiations, the present IRI results do not support Keele's interpretation that a necessary interinitiation delay is responsible for the PRP effect.

Finally, while statistical tests failed to discriminate $R_{2}$ ISI functions generated by the neutral and accuracy groups, the consistency with which intermediate effects appeared for the neutral group suggests a quantitative, 
as well as qualitative, effect of SAT demands upon $R_{2}$ performance. It is to be remembered that statistical comparisons among the three experimental groups were based upon between-subjects error. A more sensitive within-subjects test for intermediate, quantitative SAT effects may be required.

\section{REFERENCE NOTES}

1. Triggs, T. J. Capacity sharing and speeded reactions to successive signals. Technical report No. 9, 08773-23-T, 1968, Human Performance Center, University of Michigan.

2. Swensson, R. G. Cognitive deadline models in reaction time. Paper presented at Eighth Annual Mathematical Psychology Meeting, Purdue University, 1975.

3. Link, S. The random walk interpretation of response deadline results. Paper presented at Eighth Annual Mathematical Psychology Meeting, Purdue University, 1975.

\section{REFERENCES}

BoRgER, R. The refractory period and serial choice-reactions. Quarterly Joumal of Experimental Psychology, 1963, 15, 1-12.

FITTS, P. M. Cognitive aspects of information processing: III. Set for speed versus accuracy. Joumal of Experimental Psychology, 1966, 71, 849-857.

Helson, H., \& Steger, J. A. On the inhibitory effects of a second stimulus following the primary stimulus to react. Journal of Experimental Psychology, 1962, 64, 201:205.

Herman, L. M. Effects of second signals on response time to first signals under certainty and uncertainty. Journal of Experimental Psychology, 1969, 80, 106-112.

Herman, L. M., \& Kantowitz, B. H. The psychological refractory period: Only half the double-stimulation story? Psychological Bulletin, 1970, 73, 74-88.

Kanneman, D. Attention and effort. Englewood Cliffs, N.J: Prentice Hall, 1973.

Kantowitz, B. H. Double stimulation with varying response information. Journal of Experimental Psychology, 1969, 82, 347-352.

Kantowitz, B. H. Double stimulation. In B. H. Kantowitz (Ed.) Human information processing: Tutorials in performance and cognition. Hillsdale, N.J: Erlbaum, 1974. (a)

KANTowitz, B. H. Double stimulation with varying response requirements. Journal of Experimental Psychology, 1974, 103, 1092-1107. (b)

Kantowitz, B. H., \& Sanders, M. S. Partial advance information and stimulus dimensionality. Journal of Experimental Psychology, 1972, 92, 412-418.

Keele, S. W. Attention and human performance. Pacific Palisades, Calif: Goodyear, 1973.
KNIGHT, J. L., JR., \& KANTowitz, B. H. Speed-accuracy tradeoff in double stimulation: Effects on the first response. Memory \& Cognition, 1974, 2, 522-532.

Kornblum, S. Simple reaction time as a race between signal detection and time estimation: A paradigm and model. Penception \& Psychophysics, 1973, 13, 108-112.

Moray, N., \& FitTer, M. A theory and the measurement of attention. In S. Kornblum (Ed.) Attention and performance (Vol. V). New York: Academic Press, 1973.

Oltman, R. T. Fast guesses in choice reaction time. Psychonomic Science, 1966, 6, 155-156.

REYNolds, D. Effects of double stimulation: Temporary inhibition of response. Psychological Bulletin, 1964, 62, 333-347.

TAYlor, M. M., \& Creelman, C. D. PEST: Efficient estimates on probability functions. Journal of the Acoustical Society of America, 1967, 41, 782-787.

WELFORD, A. T. The "psychological refractory period" and the timing of high-speed performance-a review and a theory. British Journal of Psychology, 1952, 43, 2-19.

WELFORD, A. T. Evidence of a single-channel decision mechanism limiting performance in a serial reaction task. Quarterly Joumal of Experimental Psychology, 1959, 11, 193-210.

Yellort, J. I. Correction for fast guessing and the speedaccuracy tradeoff. Joumal of Mathematical Psychology, 1971, 8, 159-199.

\section{NOTES}

1. The Stimulus Pair variable is explained in terms of correct responses in the hope that the reader might best visualize the spatial locations of $S_{1}$ and $S_{2}$ by reference to the familiar geometry of his own hands. Note that although a particular Stimulus Pair might call for keypresses by both left- and righthand index fingers (i.e., Stimulus Pair II), the subject might produce a Response Pair, such as MM, containing one or more incorrect responses.

2. Single-stimulation data were not analyzed in this case, since the stimulus and response sets used in single stimulation were not the same as those used for $R_{1}$ in double stimulation. However, single-stimulation control data are presented in Table 1. These control points are basically similar to those obtained at ISI $=480 \mathrm{msec}$.

(Received for publication December 11, 1975; revision received April 5, 1976.) 